\title{
DISTRIBUCIÓN ESPACIAL 3D DEL VALOR-B EN EL SECTOR DEL VALLE MEDIO DEL MAGDALENA, COLOMBIA
}

\author{
3D SPATIAL DISTRIBUTION OF THE B-VALUE IN THE MIDDLE MAGDALENA VALLEY \\ SECTOR, COLOMBIA
}

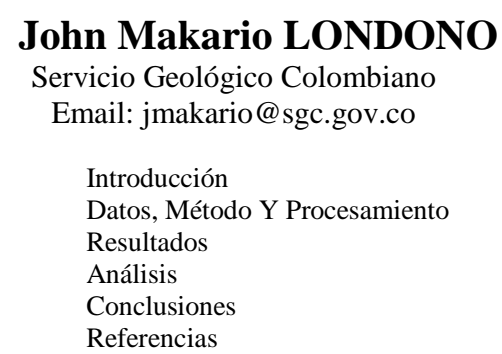

\begin{abstract}
RESUMO - Um mapeamento 3D espacial do valor b foi realizado para a região de Valle Medio del Magdalena (VMM), na Colômbia. A distribuição espacial do valor b nessa região mostrou importantes mudanças de Oeste para Leste, com baixos valores de b $(<0,6)$ para o Ocidente indicando acúmulo de estresse, valores normais a altos para o centro de VMM indicando estabilidade tectônica e normal para Baixos valores $b(<1,0)$ para o Leste indicando acúmulo de tensão, embora para o NE e SE altos valores b foram observados $(1,2-1,5)$. A Falha de Bucaramanga mostrou algumas porções com baixos valores $b(<0,7)$ e algumas outras com altos valores $b(>1,2)$, indicando possivelmente a presença de asperezas. Outras estruturas geológicas, como as falhas Curumaní, Arenas Blancas e Cimitarra, mostraram uma correlação entre os valores baixos-b $(<0,8)$ para os terremotos crustais rasos e altos valores b para os terremotos mais profundos, indicando provavelmente a liberação de tensões em profundidades superficiais nesses sistemas de falha. A placa de Nazca apresentou valores de b entre 0,8 e 1,0 até $120 \mathrm{~km}$ de profundidade. Foram observadas variações espaciais do valor b dentro da placa de Nazca, particularmente ao norte e centro da VMM, entre 10 e $30 \mathrm{~km}$ de profundidade. Nenhuma evidência de subducção profunda para a placa do Caribe foi observada neste setor da Colômbia.
\end{abstract}

Palavras-chave: Valor b, Valle Medio del Magdalena Colômbia, Placa de Nazca, Falha de Bucaramanga, Sismicidade cortical.

\begin{abstract}
A spatial 3D mapping of b-value was carried out for Valle Medio del Magdalena (VMM) region, Colombia. The spatial distribution of $b$-value in that region showed important changes from West to East, with low b-values $(<0.6)$ to the West indicating stress accumulation, normal to high values to the center of VMM indicating tectonic stability, and normal to low b-values $(<1.0)$ to the East indicating stress accumulation, although to the NE and SE high b-values were observed (1.2-1.5). Bucaramanga Fault showed some portions with low b-values $(<0.7)$ and some others with high b-values $(>1.2)$, indicating possibly the presence of asperities. Other geological structures such as Curumaní, Arenas Blancas and Cimitarra faults showed a correlation between low-b values $(<0.8)$ for shallow crustal earthquakes and high b-values for deeper earthquakes, indicating probably stress release at shallow depths in these fault systems. Nazca plate slab showed b-values between 0.8 and 1.0 up to $120 \mathrm{~km}$ depth. Spatial variations of b-value inside the Nazca plate slab were observed, particularly to the north and center of VMM, between 10 and 30km depth. No evidence of deep subduction for Caribbean plate were observed in this sector of Colombia.
\end{abstract}

Keywords: b-value, Valle Medio del Magdalena Colombia, Nazca plate, Bucaramanga Fault, cortical seismicity.

\section{INTRODUCCIÓN}

La relación frecuencia-magnitud de sismos, es una medida utilizada normalmente para determinar niveles de actividad sísmica, así como acumulación de esfuerzos (Tormann et al., 2014). Esa relación fue definida por Guttenberg \& Richter (1944, 1954) como:

$$
\log _{10} N=a-b M
$$

donde $N$ es el número acumulado de sismos con magnitud $>=M$, la constante $a$ depende del nivel de actividad en una determinada región, y la constante $b$ es la pendiente, comúnmente llamada "valor-b", la cual tiene normalmente tiene un valor de 1 , y puede variar temporalmente de acuerdo al nivel de esfuerzos aplicados en una región.
Aunque todavía no se tiene totalmente esclarecido el significado físico de los cambios en el valor $b$, estos han sido atribuidos a diferentes fenómenos físicos; valores altos de $\mathrm{b}$ $(>1.0)$ pueden estar asociados a un incremento en la heterogeneidad del material (McNutt \& Jolly, 1999), o aumento en la presión de poros (Wyss, 1973; McNutt et al, 1999), o aumento en el gradiente termal (Warren \& Latham, 1970; McNutt \& Jolly, 1999).

Por otra parte, valores bajos de b $(<1.0)$ pueden ser debidos a un aumento en la magnitud de la tensión de cizalla aplicada (Scholz, 1968; Urbancic et al., 1992), o incremento en la tensión efectiva, o disminución en la presión de poros 
(Wyss, 1973; McNutt \& Jolly, 1999).

La distribución espacial del valor b permite conocer el nivel de esfuerzos en una región determinada, en particular en zonas tectónicamente activas (Knopoff et al., 1982; Wesnousky, 1994, Öncel \& Wilson, 2004). Tal es el caso de la región de la Falla de San Andrés en los Estados Unidos, donde se han realizado estudios detallados de la variación espacial de b y su relación con la actividad de las fallas en dicha región (Tormann et al., 2014). Así mismo, se han realizado estudios de la variación espacial de $b$ en zonas de subducción, encontrando variaciones de este valor de bajos a altos en dicha zona (Katsumata, 2006).

En zonas volcánicas también ha sido utilizada la distribución espacial de $b$ (altos valores de b) para detectar zonas posiblemente asociadas a cámaras magmáticas (Wiemer \& Benoit, 1996; Wyss et al., 1997; Wiemer \& Wyss, 1997; Wiemer et al., 1998; Wyss et al., 2001; Londoño \& Rodríguez, 2014).

Este estudio se enfoca en el estudio de la distribución espacial del valor b en la región del Valle Medio del Magdalena (VMM), Colombia, con miras a detectar variaciones que puedan estar asociadas a la actividad tectónica y régimen de esfuerzos de la misma.

\section{DATOS, MÉTODO Y PROCESAMIENTO}

Existen dos métodos tradicionales para el cálculo del valor-b; el método de mínimos cuadrados pesados (ecuación 1) y el de máxima probabilidad, el cual es expresado mediante la fórmula (Aki, 1965; Utsu, 1965):

$$
b=\left(\frac{1}{[M]-M_{c}}\right) \log e
$$

Donde $[M]$ es el valor medio de todas las magnitudes dentro de los rangos seleccionados del catálogo y $M c$ la magnitud de completitud. $\log e=$ 0.4343. Para el cálculo del valor por el método de máxima probabilidad se usan todos los eventos del catálogo, el cual se crea seleccionando todos los eventos iguales o mayores que la magnitud de completitud (Mc), definida como la magnitud mínima a partir de la cual la relación lineal entre el logaritmo de la frecuencia de sismos (número acumulado) y la magnitud deja de serlo y se vuelve exponencial (ver Figura 5 más adelante).

El error $(\sigma)$ en el cálculo del valor-b está expresado por (Shi \& Bolt, 1982):

$$
\sigma(b)=2.30 b^{2} \sigma([M])
$$

donde:

$$
\sigma^{2}([M])=\frac{\sum_{i=1}^{n}\left(M_{i}-[M]\right)^{2}}{n(n-1)}
$$

en unos límites de confianza del $95 \%$ para una distribución normal. $n$ es el número de sismos, y $M$ la magnitud.

Aunque ambos métodos presentan resultados similares, se escogió el de máxima probabilidad para el análisis, por ser el método estándar del cálculo de b.

Se utilizaron 5235 sismos para el cálculo espacial de b, correspondientes al período 20142016, registrados y localizados con la red sísmica portátil temporal instalada en el VMM por parte del SGC y complementada con las estaciones cercanas de la Red Sísmica Nacional del SGC. La figura 1 muestra la localización hipocentral de los sismos usados.

Para el cálculo espacial del valor b, se utilizó la metodología de Wiemer (2001) e Wyss et al. (2001). Inicialmente se determinó la magnitud de completitud (Mc) del catálogo sísmico, la cual varió en el tiempo, y se tomó como valor promedio 1.0 (Figura 2). Después de diferentes pruebas, se dividió la zona de estudio en una cuadrícula tridimensional, con nodos cada $20 \times 20 \mathrm{~km}$ en latitud y longitud y cada $10 \mathrm{~km}$ en profundidad.

Para cada nodo se calculó el valor de b teniendo en cuenta los siguientes parámetros: número de sismos con magnitud mayor a Mc para calcular el valor $\mathrm{b}>=30$; tomando el centro de cada nodo se definió un radio de $20 \mathrm{~km}$. Todos los sismos que estuvieran localizados dentro de dicho radio se usaron para el cálculo de b en ese nodo. Aquellos nodos que no cumplían con el criterio de número mínimo de sismos fueron excluidos del cálculo del valor-b. Por último se elaboraron gráficos. El software utilizado para los cálculos fue ZMAP (Wyss et al., 2001).

\section{RESULTADOS}

La figura 3 muestra la distribución de frecuencias de la magnitud y la figura 4 muestra la distribución de frecuencias de la profundidad de los sismos. En la figura 3 se puede observar que el catálogo está completo entre las magnitudes 1 y 3 , y en la figura 4 se observa que la profundidad presenta un comportamiento bimodal o doble campana de Gauss, predominando 


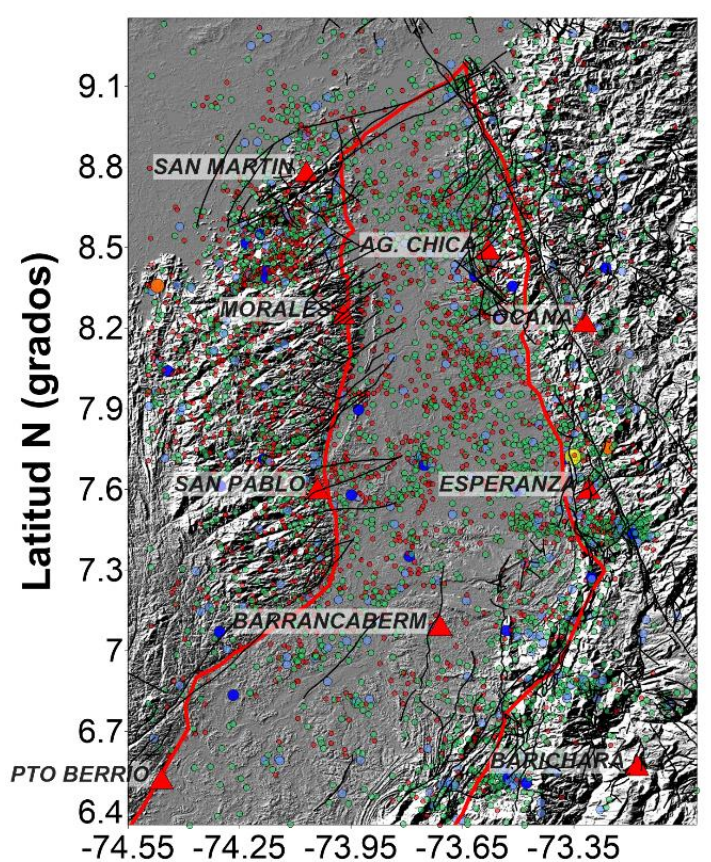

\section{Longitud W (grados)}

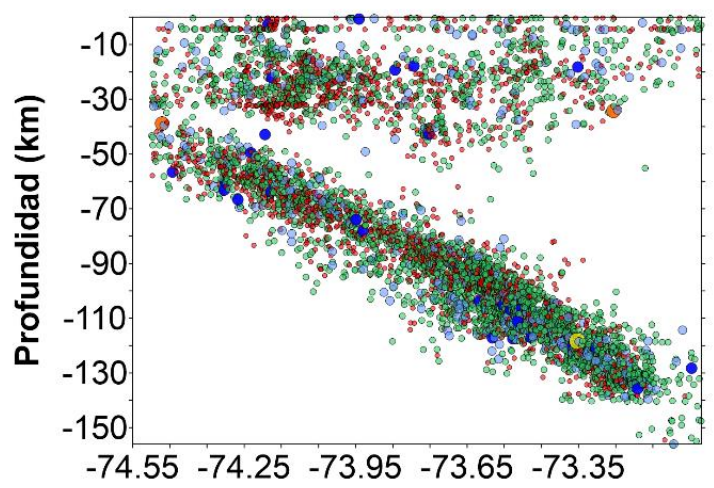

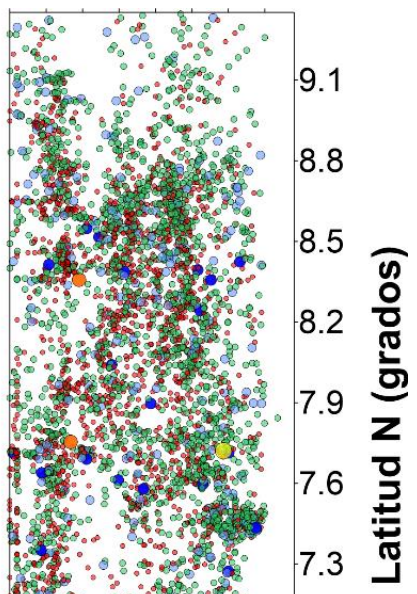

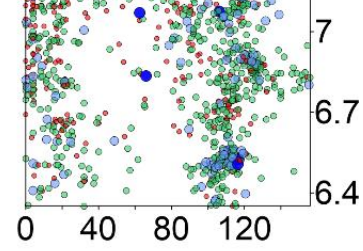

\section{Profundidad (km)}

\section{Longitud W (grados)}

Figura 1 - Área de estudio y localización hipocentral de los sismos usados para el análisis. Los triángulos rojos representan las estaciones sísmicas usadas para la determinación de los hipocentros de los sismos. La zona con línea roja gruesa representa la delimitación de la cuenca del VMM. Las líneas negras representan lineamientos y fallas geológicas.

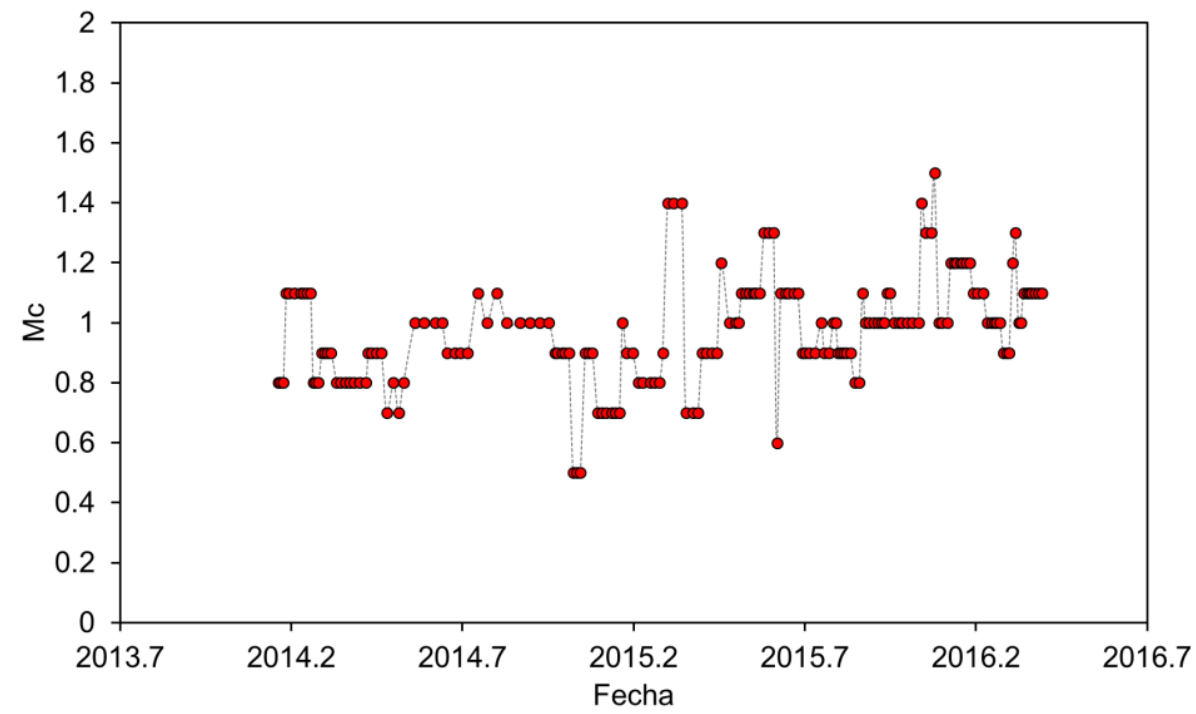

Figura 2 - Variación temporal de la magnitud de completitud (Mc) para la zona del VMM 2014-2016.

predominando las profundidades entre $20-35 \mathrm{~km}$ sismos asociados al plano de subducción, y $80-120 \mathrm{~km}$, que reflejan los sismos corticales y respectivamente (Figura 1). 
Como una primera aproximación se calculó el valor b y Mc para toda la zona, usando todos los sismos disponibles. La figura 5 muestra los resultados. Se obtuvo un valor $b$ promedio de $1.02 \pm 0.03$ y una Mc de 1.0. La Figura 6 muestra la distribución espacial en planta del valor b para la zona de estudio. Se pueden observar valores altos (b>1) en el extremo norte, extremo sur, y centro del VMM. El resto presenta valores bajos (0.6-0.8).

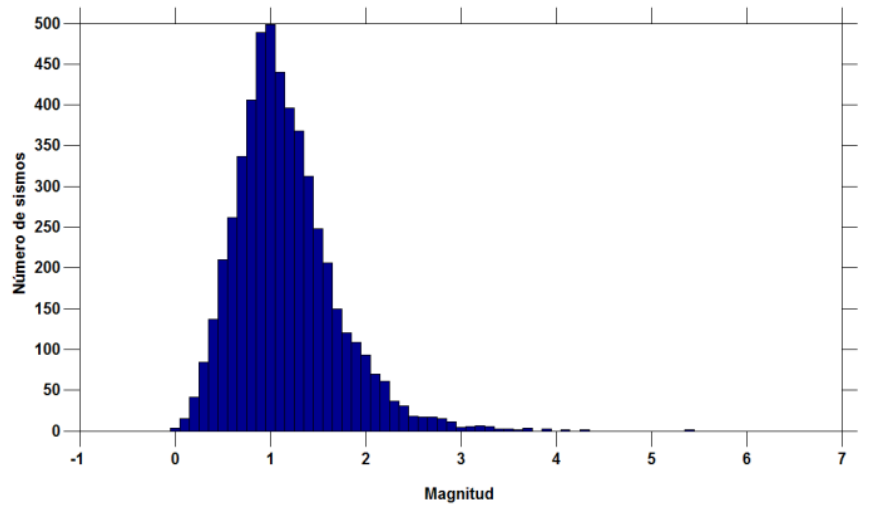

Figura 3 - Histograma de magnitud para el VMM.

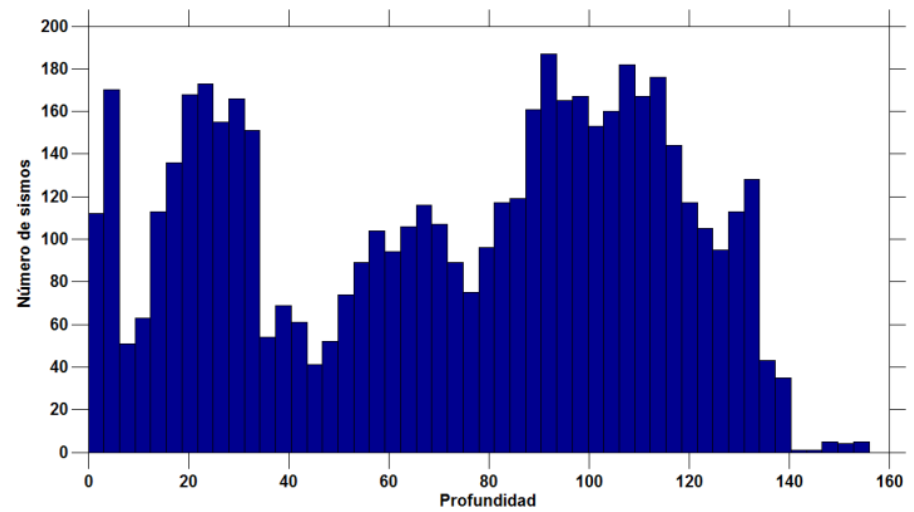

Figura 4 - Histograma de profundidades de los sismos para el VMM.

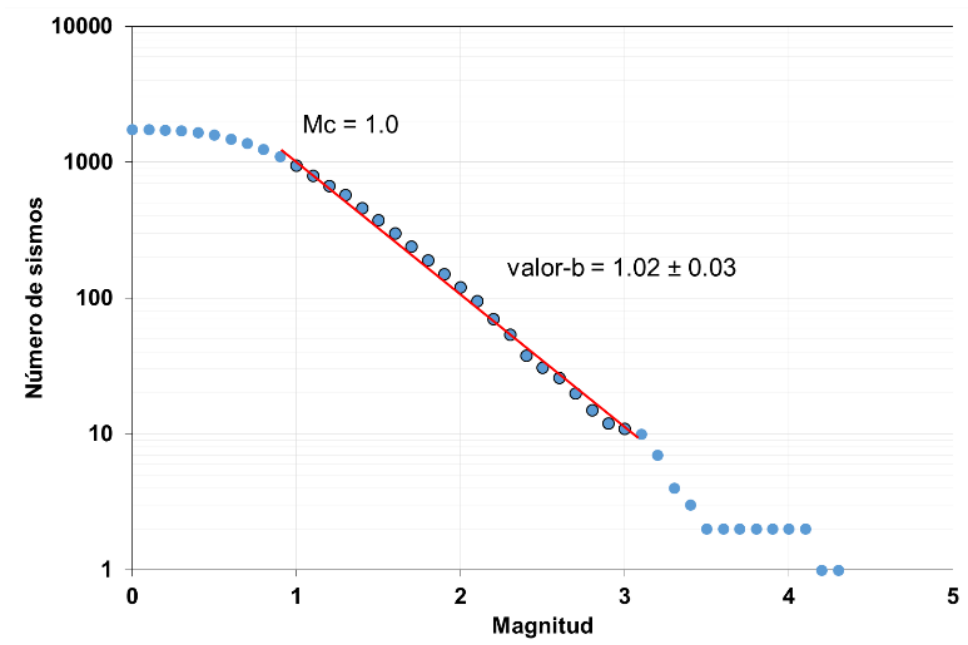

Figura 5 - Distribución de Frecuencia-magnitud para toda la zona de estudio, usando todos los sismos disponibles entre 0 y $50 \mathrm{~km}$ de profundidad. Mc=Magnitud de completitud.

Con miras a evaluar la confiabilidad de los resultados se tomaron dos zonas diferentes y se les calculó el valor b y se determinó la probabilidad de que ambos pertenezcan a la misma población, siguiendo el criterio de Utsu (1992). Una zona se localizó tomando como centro la estación San Martin, y la otra

\section{Barrancaberm (Figura 6).}

En ambas se tomó un radio hasta que estuvieran contenidos 150 sismos. La Figura 7 muestra la distribución de frecuencia-magnitud para ambas zonas. Se puede observar que ambas poblaciones son diferentes ya que la probabilidad (p) es muy baja (0.18). Con esta prueba, se 
garantiza que los valores de b obtenidos son confiables, y que las variaciones espaciales que existan son debidas más probablemente a parámetros físicos que a artifactos matemáticos o estadísticos.

La figura 8 muestra un perfil $\mathrm{EW}$ de la distribución espacial de b, pasando por la latitud $8^{\circ}$ con un ancho de $50 \mathrm{~km}$ a cada lado de dicha
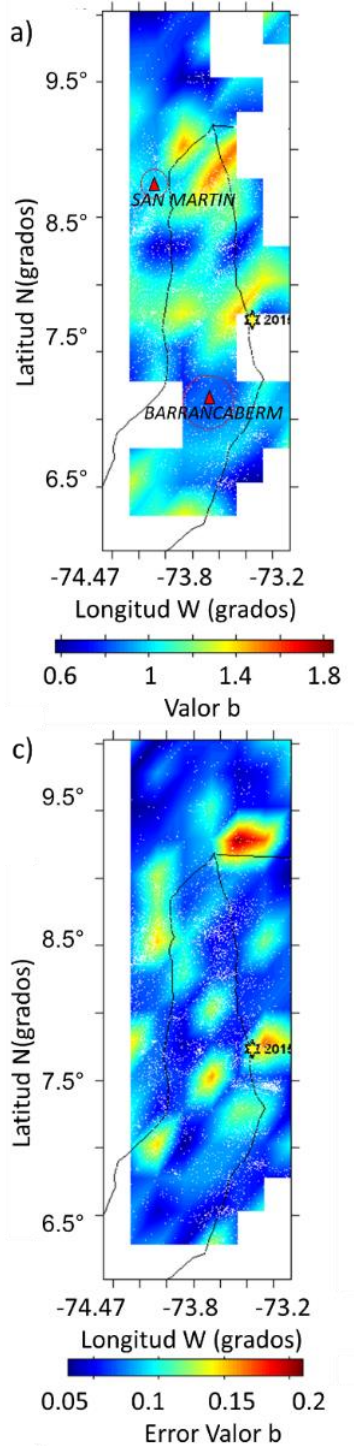

latitud.

En general se observan valores bajos de b $(<0.7 \pm 0.15)$. Adicionalmente, se elaboraron otros dos perfiles a $7^{\circ}$ y $9^{\circ}$ de latitud, con $50 \mathrm{~km}$ a cada lado, para observar la variación espacial de $b$ (Figura 9), donde se observan valores bajos de $\mathrm{b}(<0.8)$ hacia el $\mathrm{W}$ y altos $(>1.3)$ hacia el $\mathrm{S}$ a mayores profundidades $(>60 \mathrm{~km})$.
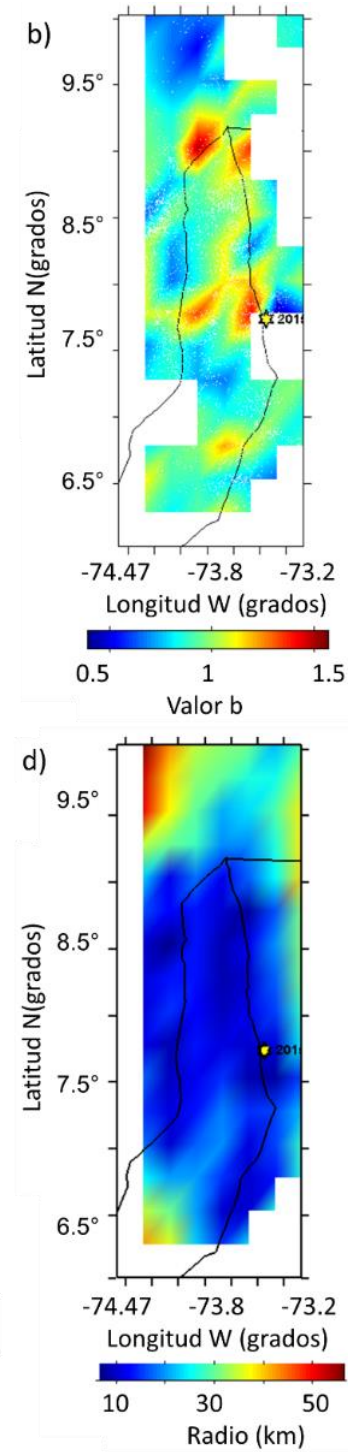

Figura 6 - Distribución espacial en planta del valor b para para el VMM (línea negra solida) usando el método de máxima probabilidad a) y mínimos cuadrados pesados b), error en el cálculo de b c) y resolución espacial d) de b (kilómetros necesarios para encontrar 50 sismos en cada nodo). Los puntos blancos representan los epicentros de los sismos usados. La estrella representa el sitio de localización hipocentral del sismo con mayor magnitud en el período estudiado. Los triángulos representan dos estaciones sísmicas y los círculos rojos punteados la zona escogida para evaluar el valor b en ambas estaciones (Figura 7).

Por otra parte, se elaboraron perfiles NS, el principal pasando por los $74^{\circ}$ de longitud y otro adicional a $\operatorname{los} 73.5^{\circ}$ de longitud (Figura10), donde se observan valores bajos $(<0.8)$ hasta profundidades de $50 \mathrm{~km}$, y mayores valores $(<1.0)$ a mayores profundidades.

Así mismo, se calculó la variación del valor b con la profundidad. Para ello se calculó el valor b para grupos de 150 sismos, con traslape cada 5 sismos, tomando como criterio de traslape su profundidad.La figura 11 muestra los resultados.

Se observan cambios importantes en el valor $\mathrm{b}$ a varias profundidades; a los $5 \mathrm{~km}(0.8 \pm 0.15)$, a los $10 \mathrm{~km}(1.2 \pm 0.15)$; a los $22 \mathrm{Km}(0.76 \pm 0.09)$, a los $25 \mathrm{~km}(1.35 \pm 0.15)$, a los $28 \mathrm{~km}(0.9 \pm 0.07)$, a los $30 \mathrm{~km}(1.2 \pm 0.16)$ y a $\operatorname{los} 50 \mathrm{~km}(0.7 \pm 0.08)$. 


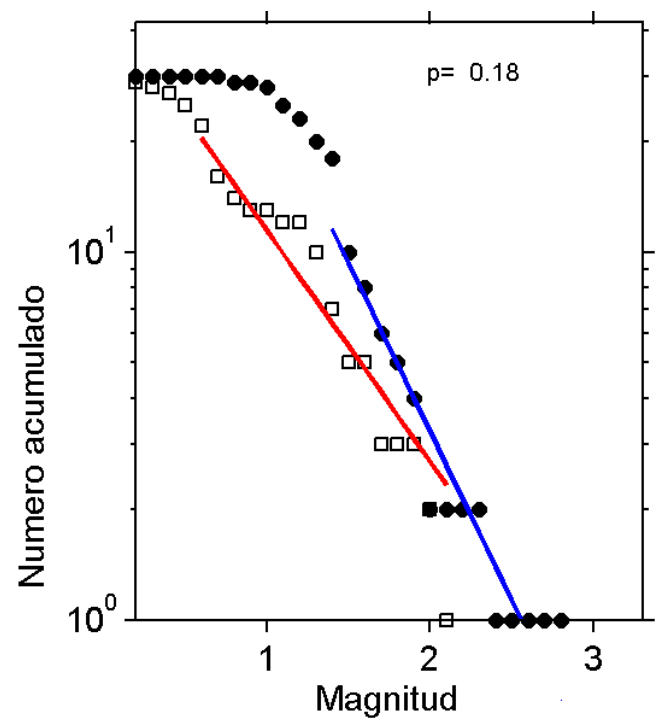

b-value ( $w$ LS, M >= 0.6): $-0.628+/-0.16$, a-value = 1

b-value ( $w$ LS, $M>=1.4$ ): $-0.916+/-0.07$, a-value $=2$

Figura 7 - Comparación de valores de b para dos zonas diferentes. La línea negra corresponde a la zona de San Martin y la zona roja a Barrancaberm (ver Figura 6).
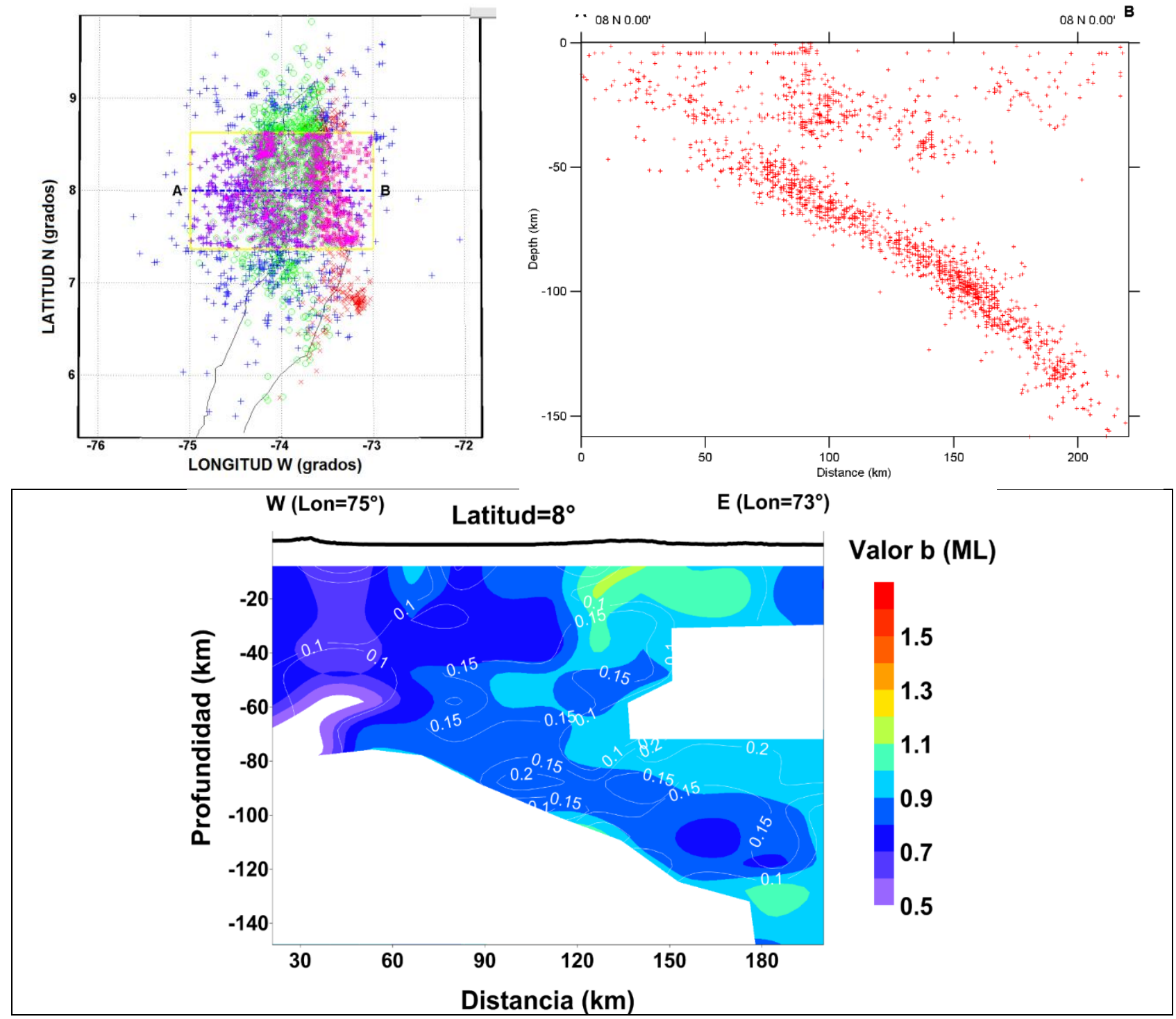

Figura 8 - Perfil EW con distribución espacial del valor b para el VMM. En la parte superior se muestran los sismos usados para el cálculo de b. Las líneas de contorno blancas representan el error estándar de b. 


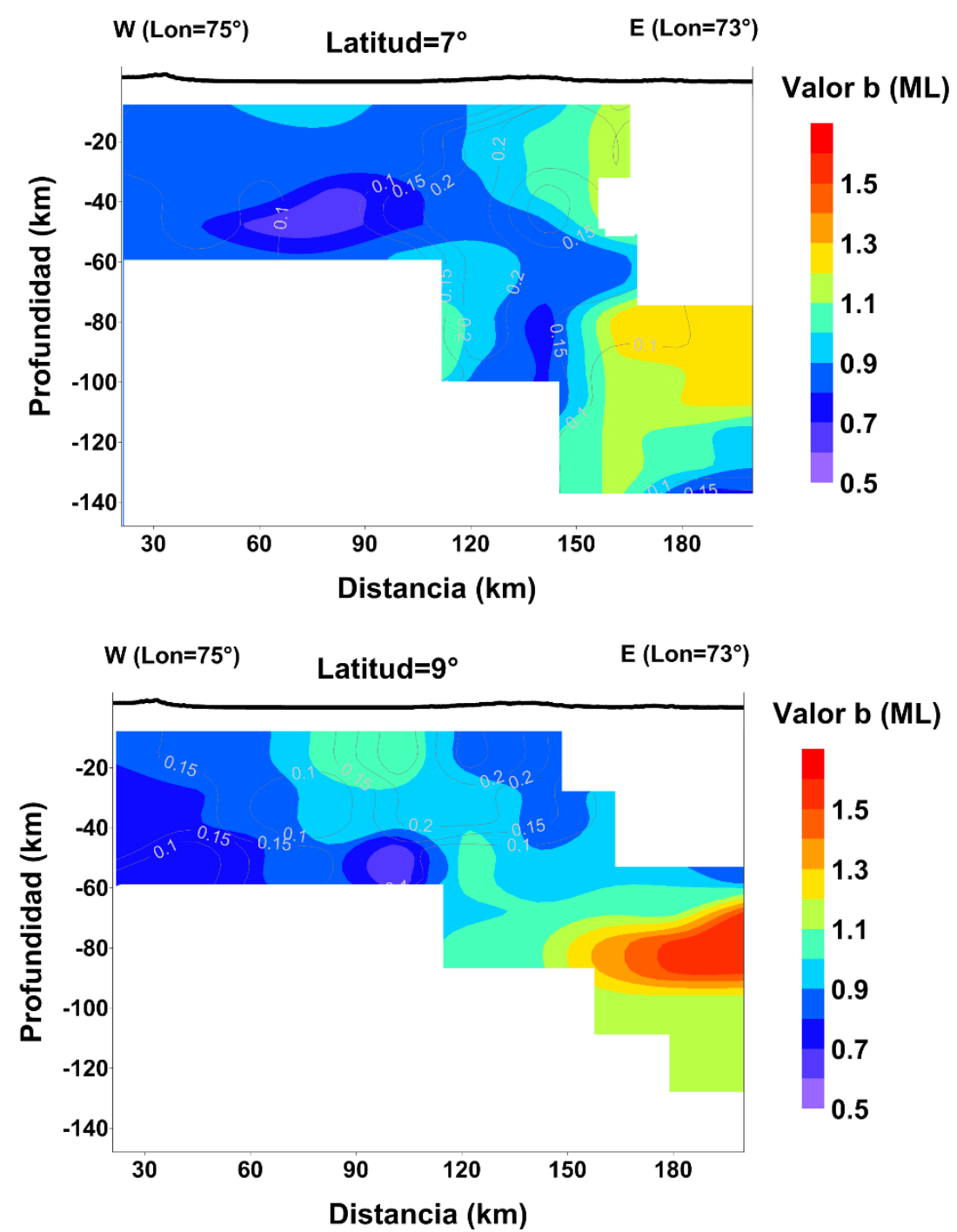

Figura 9 - Perfiles EW a $7^{\circ}$ y $9^{\circ}$ de latitud con distribución espacial del valor b para el VMM. Las líneas de contorno representan el error estándar de b. El ancho de la zona corresponde a 50km a partir de las latitudes $7^{\circ}$ y $9^{\circ} \mathrm{N}$, respectivamente.

De acuerdo con los resultados, se puede decir que la zona del VMM se encuentra sometida a esfuerzos en la parte $\mathrm{W}$, ya que presenta valores de $b$ bajos $(0.5-0.8)$, mientras que la zona plana que correspondería a la cuenca, presenta valores normales (0.9-1.1). En la zona E, se destacan dos rangos de valores de b; hacia el NE y SE de la zona, predominan valores altos de b (1.2-1.5 o mayores) y hacia el centro $\mathrm{E}$, predominan valores normales a bajos (0.9-1.1).

Esta distribución de valores de $b$, permite inferir algunos parámetros físicos que pueden estar causando las variaciones espaciales de b, como son, un aumento en la presión de poros, es decir, bajo niveles de estrés en la zonas NE y SE a profundidades entre 70 y $110 \mathrm{~km}$ aproximadamente, mientras que en la Serranía de San Lucas hacia el $\mathrm{W}$, predominaría baja presión de poros entre 0 y $60 \mathrm{~km}$ de profundidad aproximada.
Esta configuración de valores b, podría indicar que la zona central del VMM estaría sometida a esfuerzos que provienen del $\mathrm{W}$ y del $\mathrm{E}$.

Por otra parte, es interesante la variación del valor $\mathrm{b}$ con la profundidad para la zona del VMM; estos cambios podrían estar indicando variaciones en la litología o al menos en los niveles de esfuerzos o presión de poros para las diferentes profundidades de la zona del VMM. Es de destacar que los valores de b a $0 \mathrm{~km}$ de profundidad son muy similares a aquellos a $50 \mathrm{~km}$ de profundidad.

Con respecto a las estructuras geológicas, en la figura 8 , latitud $=8^{\circ} \mathrm{N}$, entre 130 y $150 \mathrm{~km}$ desde el inicio del perfil EW, entre 0 y $30 \mathrm{~km}$ de profundidad se aprecian valores más altos de $\mathrm{b}$ (09-1.1) que las zonas circundantes (0.7-0.8); esta zona corresponde al trazo de la Falla de Bucaramanga, lo que podría indicar que ésta 

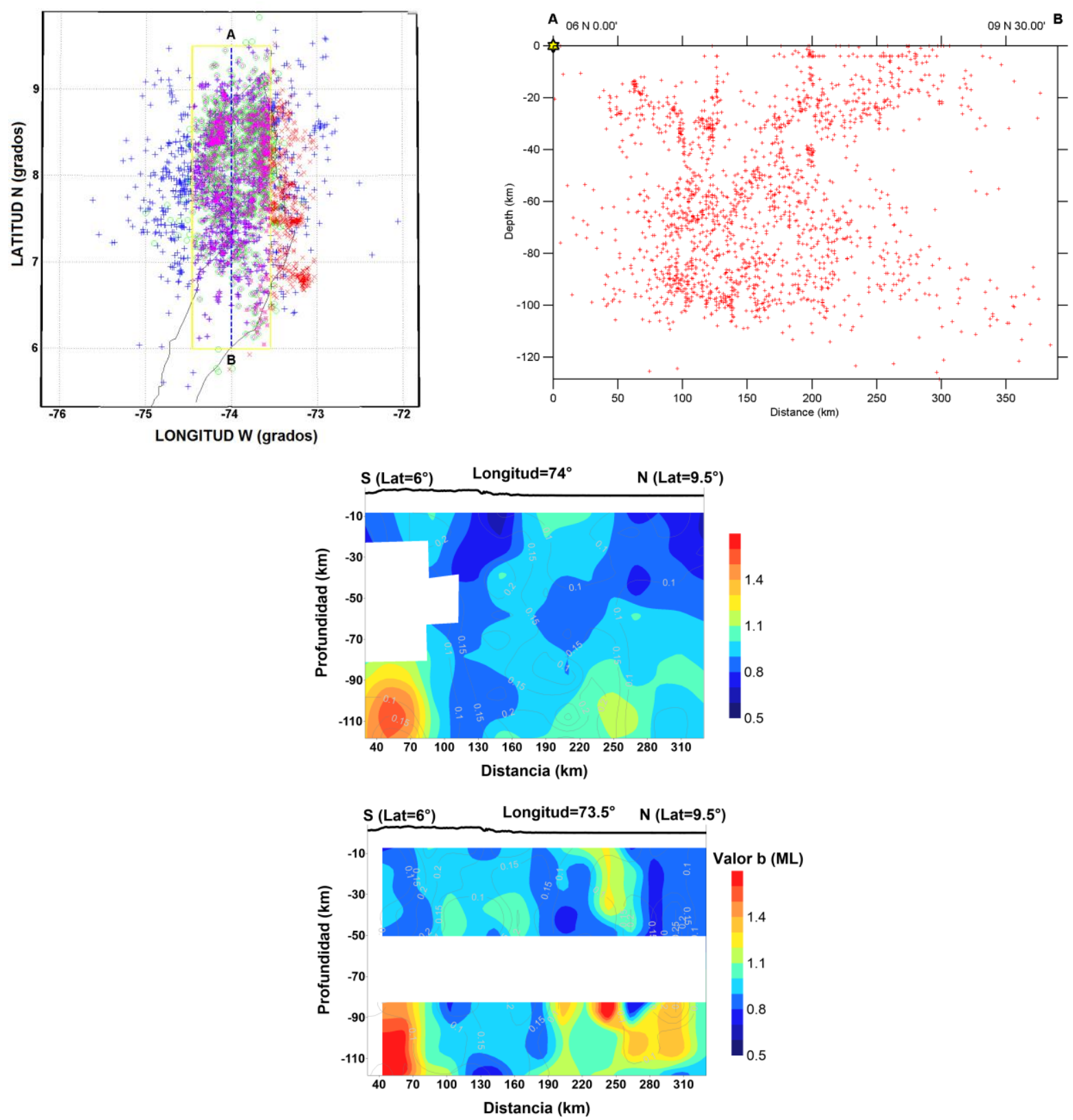

Figura 10 - Perfiles NS de la distribución espacial de b a $74^{\circ}$ y $73.5^{\circ}$ de latitud para la zona del VMM. En la parte superior se muestran los sismos usados para el cálculo. Las líneas de contornos representan los errores estándar de b. El ancho de la zona corresponde a $50 \mathrm{~km}$ a partir de las longitudes $74^{\circ}$ y $73.5^{\circ} \mathrm{W}$, respectivamente.

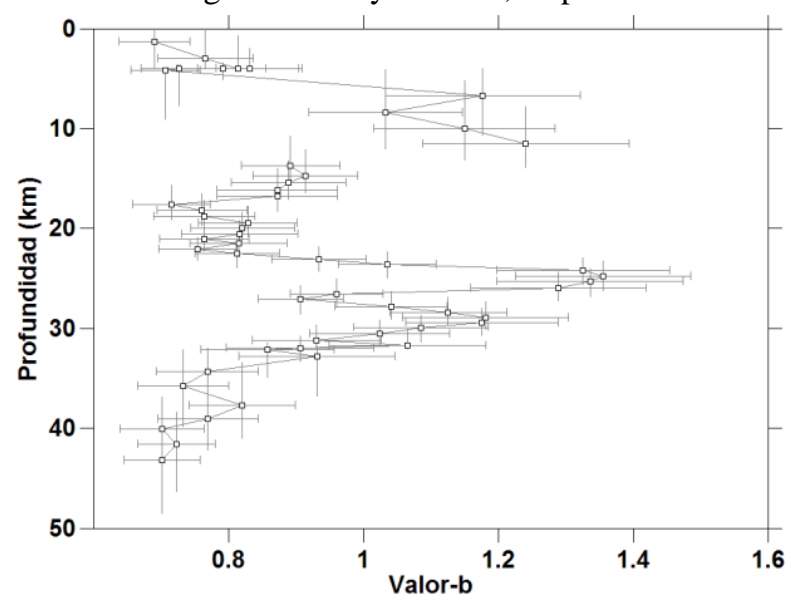

Figura 11 - Variación del valor b con la profundidad entre las latitudes $6^{\circ}-10^{\circ} \mathrm{N}$ para la zona del VMM entre 0 y $50 \mathrm{~km}$ de profundidad. Las barras horizontales representan el error estándar de b y las barras verticales representan el rango de profundidades usados para obtener 150 sismos necesarios para el cálculo de cada valor de b. El traslape entre cada punto fue de 5 sismos. 

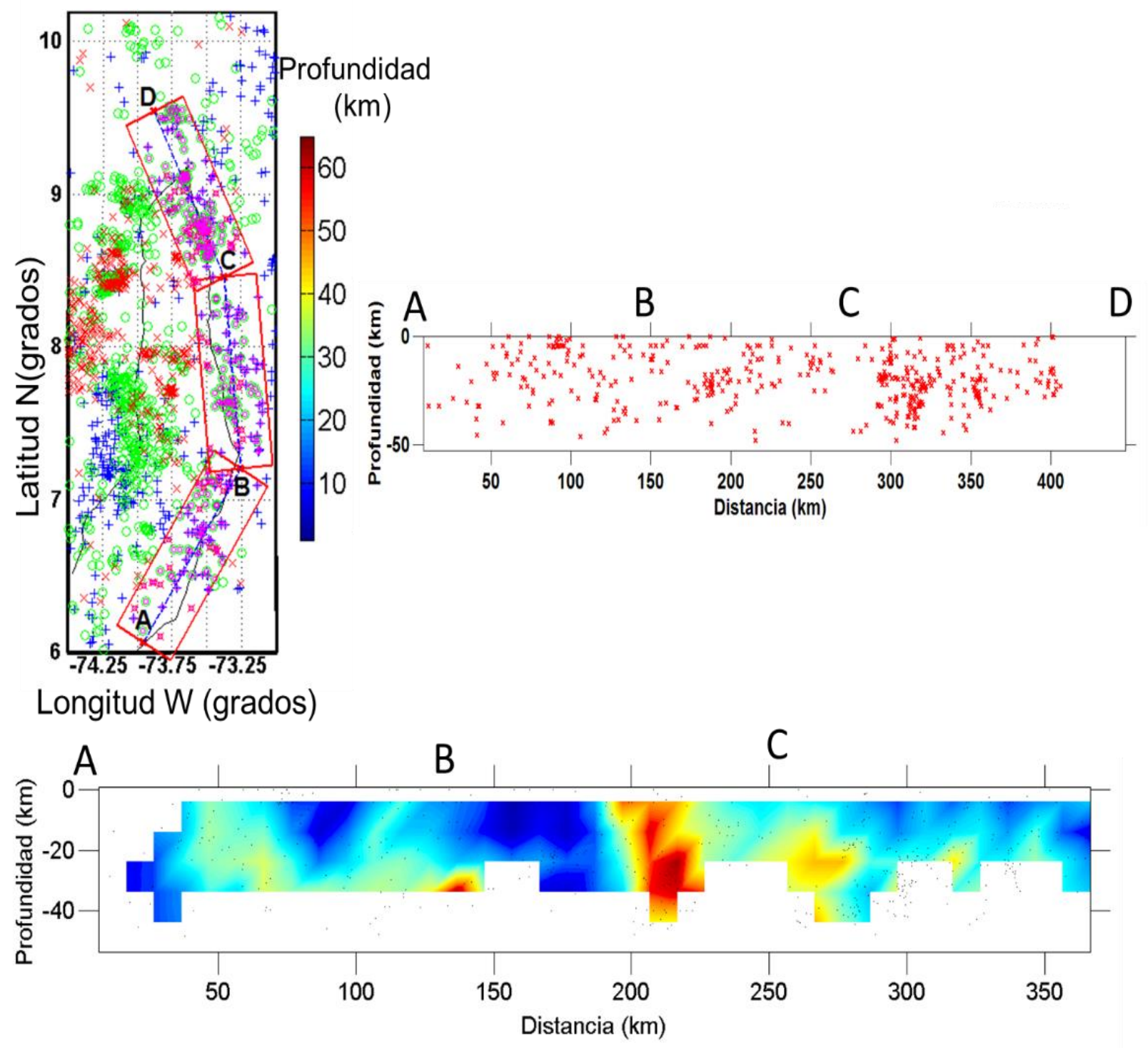

valor $\mathrm{b}$
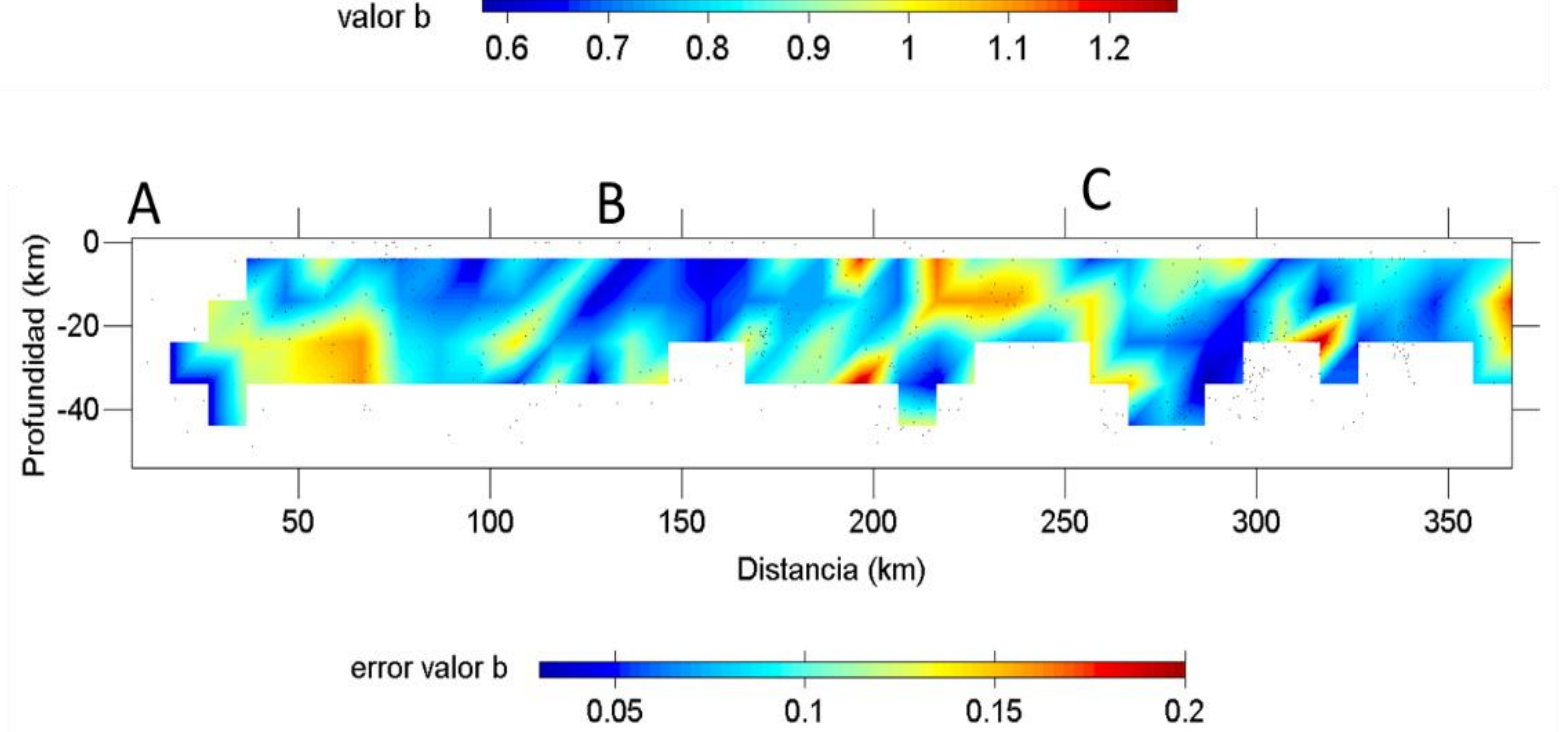

Figura 12 - Cálculo del valor b en la zona de la Falla Bucaramanga. Se muestran la vista en planta y perfil de los sismos (arriba), valor b por el método Máxima probabilidad (centro) y error del valor b (abajo). Los círculos encerrados en los rectángulos rojos representan los sismos usados para el cálculo de b. El rectángulo tiene un ancho de $100 \mathrm{~km}(50 \mathrm{~km}$ a cada lado del perfil $\mathrm{ABCD})$. 
zona de falla penetra hasta esas profundidades, y que se encontraría posiblemente en un proceso de liberación de esfuerzos, mientras que las zonas circundantes a ella estarían sometida a mayores esfuerzos. Para observar en más detalle la zona de la Falla Bucaramanga, se realizó una sección siguiendo el límite de la cuenca del VMM en la parte Este, con un ancho de $50 \mathrm{~km}$ a cada lado del perfil y se calculó el valor b, en un retícula de 10x10x10km, con mínimo 30 sismos por nodo. La figura 12 muestra los resultados.

Se puede evidenciar en más detalle tramos de la falla con mayor acumulación de esfuerzos

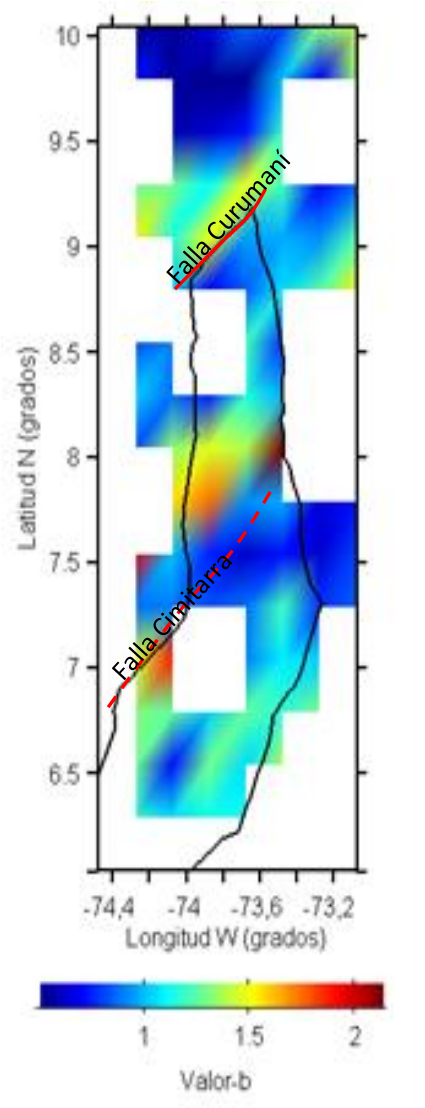

$(b<0.7)$ mientras que otros presentan liberación de los mismos ( $b>1.2)$.

Esta distribución espacial de los valores de b en la zona de la Falla Bucaramanga, sugiere la posibilidad de la existencia de barreras o asperezas (bajos valores de b) que podrían ser potenciales zonas de ruptura en un futuro y generar sismos con magnitudes mui considerables. Adicionalmente, se realizó el cálculo del valor-b solo para la zona cortical $(0-50 \mathrm{~km})$ con el fin de observar más en detalle las variaciones espaciales de dicho valor, usando una retícula de 10x10x10km en planta, con mínimo 30 sismos por nodo (Figura 13).

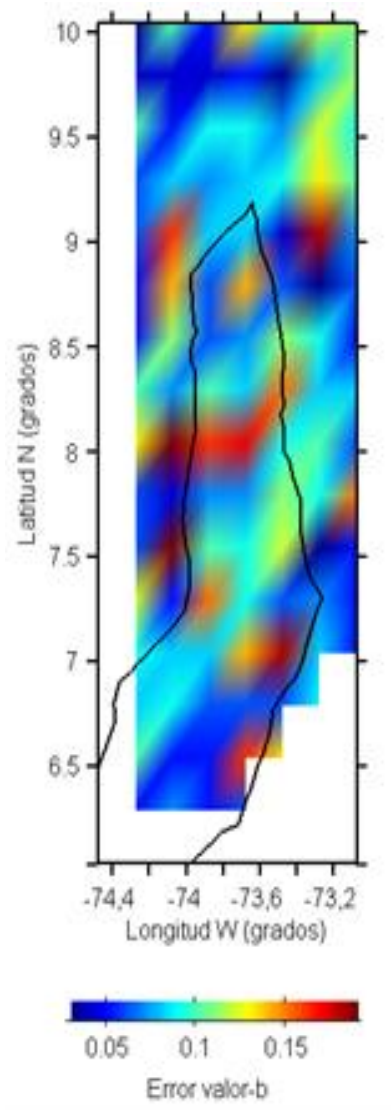

Figura 13 - Distribución espacial en planta del valor-b (izquierda) y error estándar (derecha), usando el método de máxima probabilidad para sismos corticales $(0-50 \mathrm{~km})$ en una retícula de 10x10x10km. Las líneas rojas representan fallas geológicas.

Así mismo se calculó el valor-b en profundidad para todos los sismos, tanto corticales como del manto, en un perfil NS usando el mismo tamaño de retícula de $10 \mathrm{x} 10 \mathrm{x}$ 10km y mínimo 30 sismos por nodo (Figura 14).

En las figuras 13 y 14 es posible observar algunas estructuras geológicas de la zona de estudio, como son la Falla Curumaní y Arenas Blancas al norte y Cimitarra en la parte central del VMM, las cuales presentan valores normales $\mathrm{a}$ altos de $\mathrm{b}(>1.5)$ en planta y valores bajos $(0.8)$ en profundidades corticales, lo que podría explicarse por liberación de esfuerzos en esta fallas, las cuales posiblemente no generen sismos fuertes de carácter superficial.

Estos valores podrían estar indicando zonas de acumulación de esfuerzos en la parte superficial de la corteza $(10-20 \mathrm{~km})$, mientras que en la parte profunda $(40-80 \mathrm{~km})$ podrían representar áreas de baja presión de poros o de mayor temperatura asociadas posiblemente a las zonas de generación de estas fallas en la corteza inferior o manto superior (Figuras 14 e 15).

Así mismo, es posible delimitar el slab o losa de subducción de la placa Caribe, en los perfiles EW (Figura 9). 

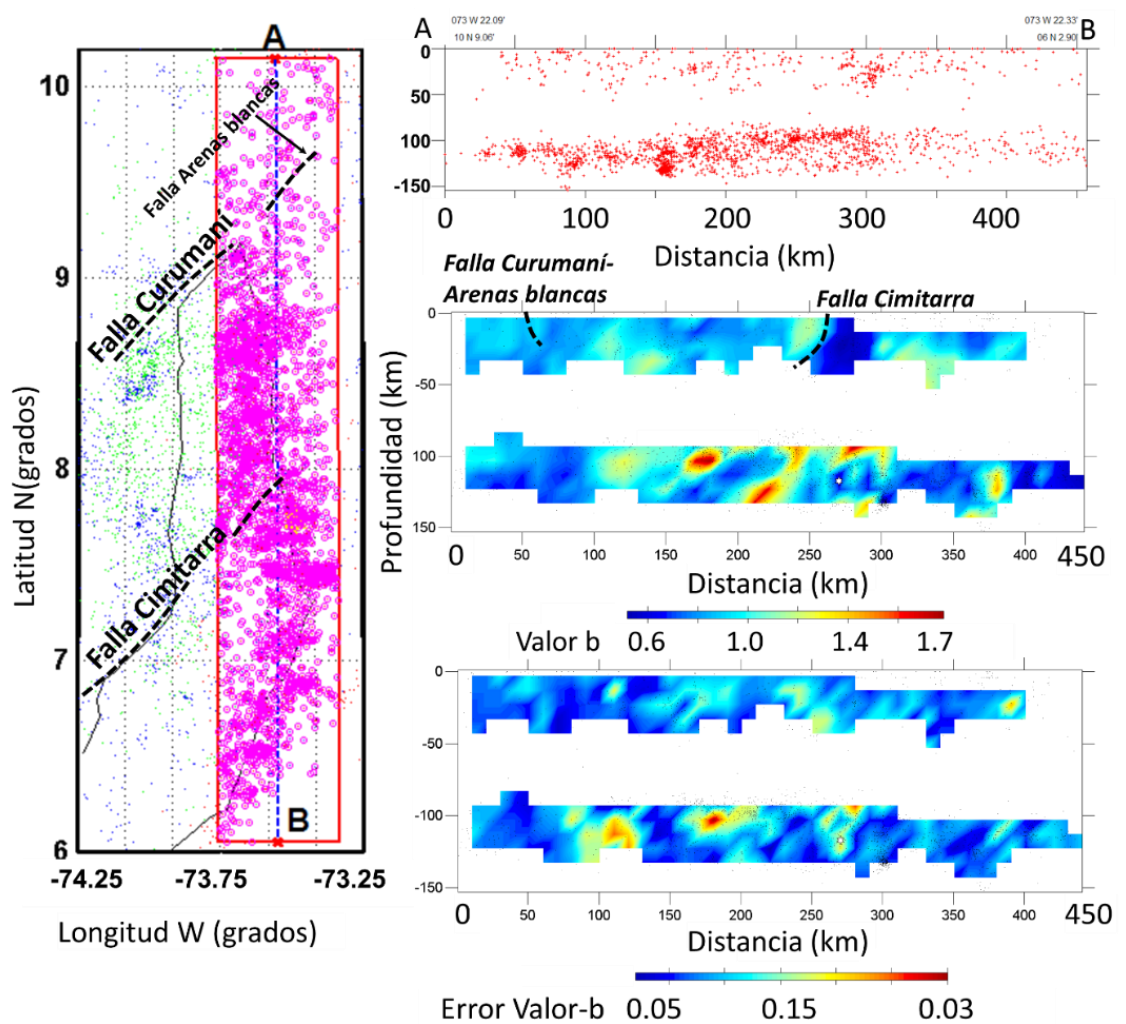

Figura 14. Distribución espacial en perfil NS (Lon $=73.45^{\circ} \mathrm{W}$ ) del valor-b y su error usando el método de máxima probabilidad para una retícula de 10x10x10km. Los círculos encerrados entre el rectángulo rojo representan los sismos usados para el cálculo de b. El rectángulo tiene un ancho de $140 \mathrm{~km}$ (70km a cada lado del perfil AB) Algunas estructuras geológicas se representan con líneas punteadas. Los valores de b se presentan interpolados.
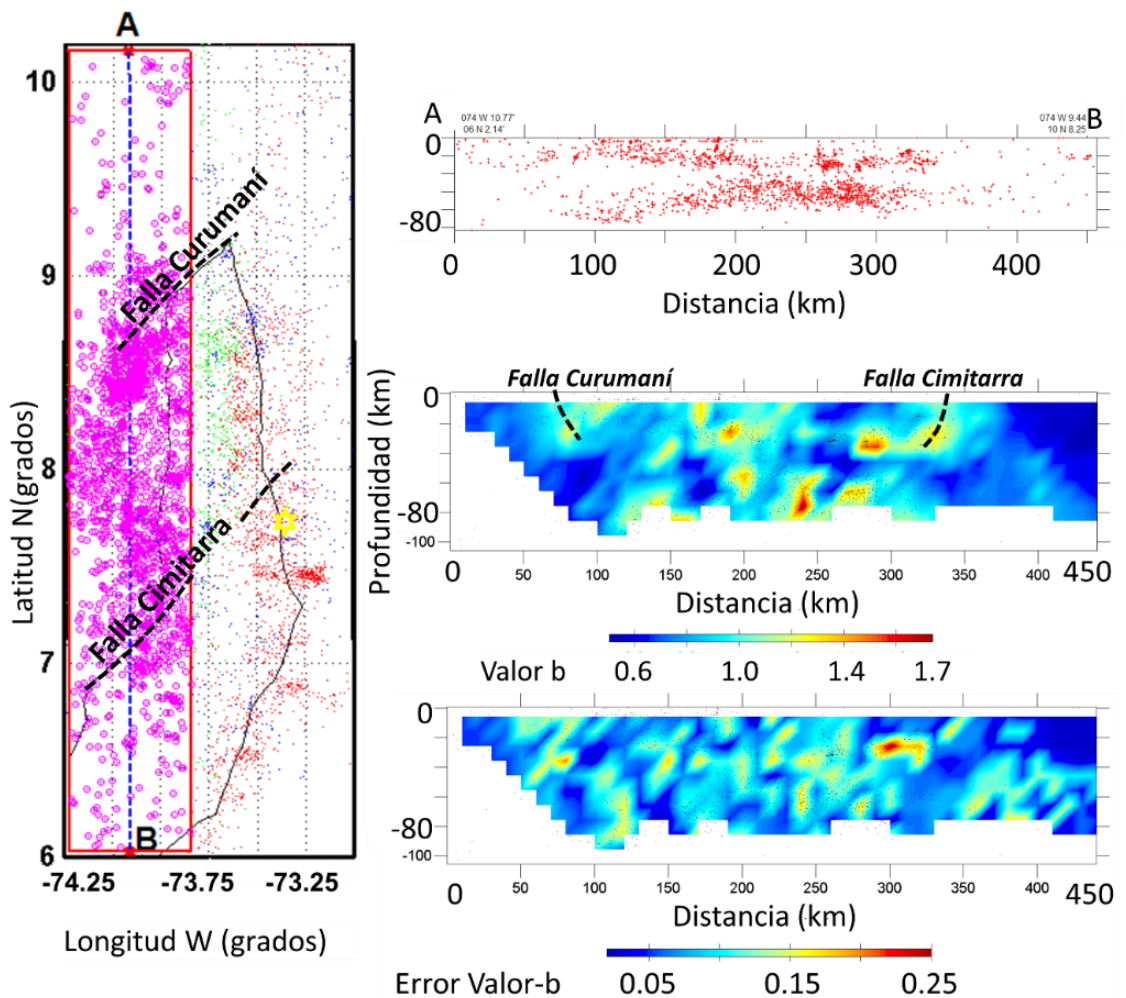

Figura 15. Distribución espacial en perfil NS $\left(\mathrm{Lon}=73.9^{\circ} \mathrm{W}\right)$ del valor-b usando el método de máxima probabilidad para una retícula de 10x10x10km. Los círculos encerrados entre el rectángulo rojo representan los sismos usados para el cálculo de b. El rectángulo tiene un ancho de $140 \mathrm{~km}$ (70km a cada lado del perfil AB) Algunas estructuras geológicas se representan con líneas punteadas. Los valores de b se presentan interpolados. 
Allí se observa que los valores de b varían entre 0.6 y 0.9 , los cuales son muy similares a los encontrados en el slab pacífico en la zona de subducción de Japón (Katsumata, 2006), mientras que hacia la esquina NE del VMM a una profundidad de $80 \mathrm{~km}$ se observa una zona con un alto valor de $b(>1.4)$, que podría estar indicando una zona con una gradiente geotérmico mayor.

Adicionalmente, el frente del slab o losa de subducción de esta placa se observa en los cortes NS (Figura 10), el cual presenta variaciones importantes de los valores de $\mathrm{b}$; en particular valores altos $(\mathrm{b}>1.3)$ hacia el sur $\left(\right.$ Lat $\left.6^{\circ} \mathrm{N}\right)$ entre 90 y $110 \mathrm{~km}$ de profundidad, y valores bajos $(\mathrm{b}<0.7)$ hacia el norte (Lat $9.5^{\circ} \mathrm{N}$ ) y parte central (Lat $7.5^{\circ} \mathrm{N}$ ) de la zona entre 20 y $30 \mathrm{~km}$ de profundidad.

La Figura 16 muestra un esquema de los posibles límites de dichas losas de subducción. Los valores altos de $b$, podrían estar asociados a porciones del slab con mayores temperaturas o mayor deshidratación (Wiemer \& Benoit, 1996; Wyss et al, 2001; Katsumata, 2006).

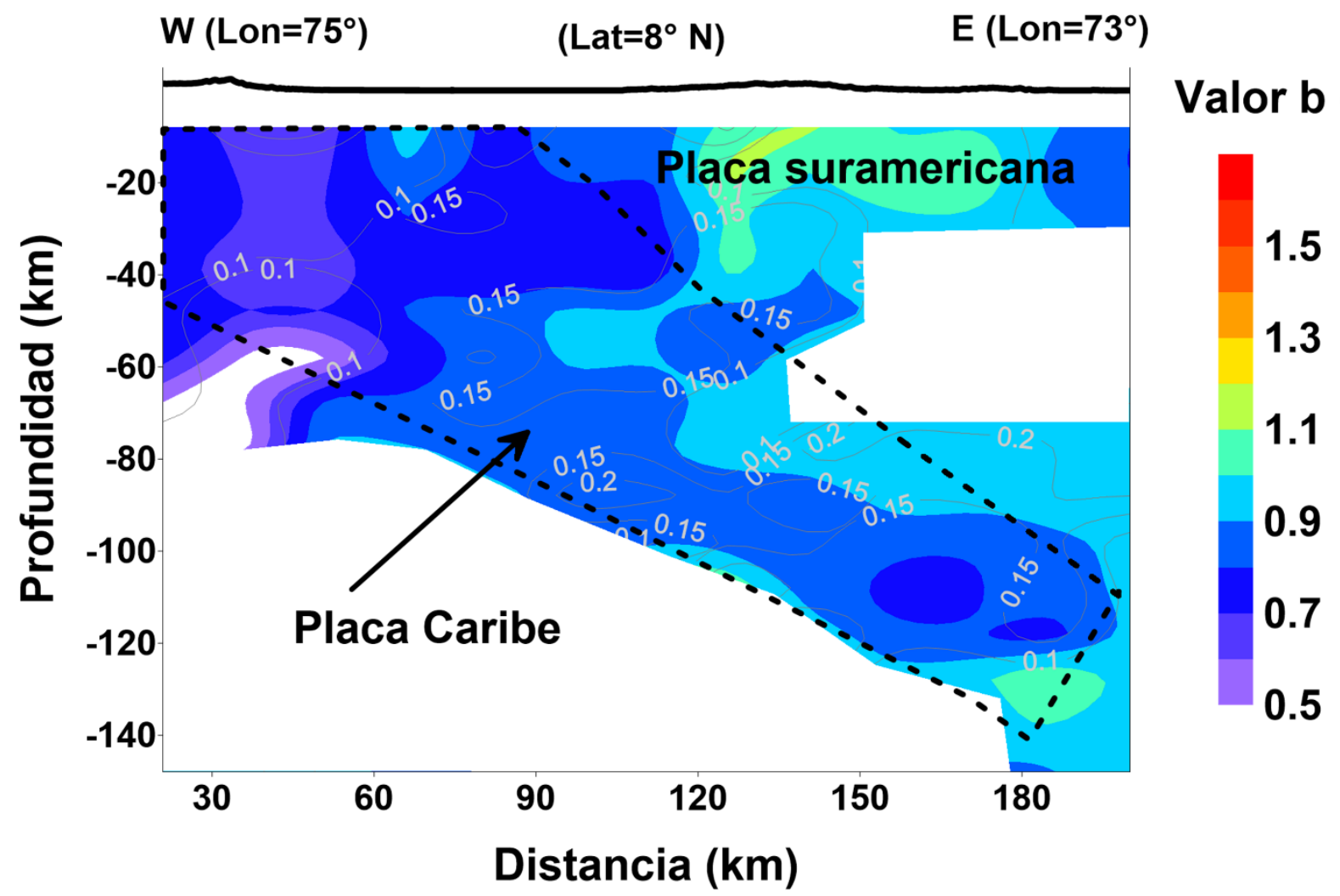

Figura 16 - Esquema de los posibles límites de las losas de subducción a partir de la distribución espacial del valor b en la zona del VMM.

\section{CONCLUSIONES}

El VMM se caracteriza, en general, por valores bajos de b (0.7- 0.8), con algunas zonas con valores altos, como la parte del extremo norte, en la parte central y en el extremo sur (>1.3).

La zona $\mathrm{W}$ se caracteriza por valores bajos, mientras que la zona $\mathrm{E}$ se presentan valores un poco mayores. Al parecer el VMM se encuentra en un estado de acumulación de esfuerzos en la parte W. Algunas estructuras como las fallas Curumaní y Cimitarra se caracterizan por presentar valores altos de $b$ hacia zonas profundas $(>30-40 \mathrm{~km})$, y valores relativamente bajos $(<0.8)$ cerca de la superficie. La zona de la Falla Bucaramanga se caracteriza por valores altos de b, lo que podría estar indicando liberación de esfuerzos, en lugar de acumulación en dicha falla.

\section{REFERÊNCIAS}

AKI, K. Maximum Likelihood estimate of $b$ in the formula Log $\mathrm{N}=\mathrm{a}-\mathrm{bM}$ and its confidence limits. Bulletin of Earthquake Research Institute, Tokyo Univ., v. 43, p. 237-239, 1965. GUTTENBERG, R. \& RICHTER, C.F. Frequency of earthquakes in California. Bulletin of the Seismological Society of America, v. 34, p. 185-188, 1944.
GUTENBERG, B. \& RICHTER, C.F. Seismicity of the Earth, 2nd ed., Princeton University, Princeton, New Jersey, 1954. KATSUMATA, K. Imaging the high b-value anomalies within the subducting Pacific plate in the Hokkaido corner. Earth Planets Space, v. 58, p. 49-52, 2006.

KNOPOFF, L.; KAGAN, K.K.; KNOPOFF, R. b-values for 
foreshocks and aftershocks in real and simulated earthquake sequences. Bulletin of the Seismological Society of America, v. 72, p. 1663-1676, 1982.

LONDOÑO, J.M. \& RODRÍGUEZ, S.P. Mapeo tridimensional del valor b en el volcán Nevado del Ruiz. Revista Ventana Informática, v. 29, p. 24-137, 2014.

MCNUTT, R.S. \& JOLLY, D.A. Seismicity at the volcanoes of Katmai National Park, Alaska. Journal of Volcanology and Geothermal Research, v. 93, p. 173-190, 1999.

ÖNCEL, A.O. \& WILSON, T. Correlation of seismotectonic variables and GPS strain measurements in western Turkey. Journal of Geophysical Research, v. 109, B11306, doi:10. 1029/2004JB003101, 2004.

SCHOLZ, C.H. Microfracturing and the inelastic deformation of rock in compression, Journal of Geophysical Research, v. 73 , n. 4, p. 1417-1432, doi: 10.1029/JB073i004p01417, 1968.

SHI, Y. \& BOLT, B. The standard error of the magnitude frecuency $b$ value. Bulletin of the Seismological Society of America., v. 72, p. 1677-1687, 1982.

TORMANN, T.; WIEMER, S.; MIGNAN, A. Systematic survey of high-resolution $\mathrm{b}$ value imaging along Californian faults: inference on asperities. Journal of Geophysical Research Solid Earth, v.119, p. 2029-2054, doi:10.1002/2013JB 010867, 2014.

URBANCIC, T.I.; TRIFU, CI.; LONG, J.M. YOUNG, P. Spacetime correlations of $b$ values with stress release. Pure and Applied Geophysics, p. 139-449. doi:10.1007/BF00879946, 1992.

UTSU, T. A method for determining the value $\mathrm{b}$ in formula Log $\mathrm{N}=\mathrm{a}-\mathrm{bM}$ showing the magnitude-frequency relation for earthquakes. Geophysical Bulletin. Hokkaido Univ, v. 13, p. 99-103, 1965

UTSU, T. On seismicity. In: Report of the Joint Research Institute for Statistical Mathematics, Institute for Statistical Mathematics, Tokyo. p.139 - 157. 1992.

VAN DER HILST, R. \& MANN, P. Tectonic implications of tomography images of subducted lithosphere beneath Northwestern South America. Geology, v. 22, p. 451-454, 1994.
WARREN, N.W. \& LATHAM, G.V. An experimental study of thermally induced microfracturing and its relation to volcanic seismicity. Journal of Geophysical Research., v. 75, p. 44554464, 1970.

WESNOUSKY, S.G. The Gutenberg-Richter or characteristic earthquake distribution, Which is it? Bulletin of the Seismological Society of America, v. 84, p. 1941- 1959, 1994. WIEMER, S. \& BENOIT, J. Mapping the b value anomaly at 100 $\mathrm{km}$ depth in the Alaska and New Zealand subduction zones. Geophysical Research Letters, v. 23, n.13, p. 1557-1560, 1996.

WIEMER, S. \& WYSS, M. Mapping the frequency-magnitude distribution in asperities: An improved technique to calculate recurrence times? Journal of Geophysical Research, v. 102, p. 15115-15128, 1997.

WIEMER, S.; McNUTT, S.R.; WYSS, M. Temporal and threedimensional spatial analysis of the frequency-magnitude distribution near Long-Valley caldera, California. Geophysical Journal International, 134, 409-421. 1998.

WYSS, M. Towards a physical understanding of the earthquake frecuency distribution. Geophysical Journal of the Royal Astronomical Society, v. 31, p. 341-359, 1973.

WYSS, M.; SHIMASAKI, K.; WIEMER, S. Mapping acitve magma chambers by $b$ values beneath the off-Ito volcano Japan. Journal of Geophysical Research, v. 102, p. 20413-20422, 1997.

WYSS, M.K.; KLEIN, F.; NAGAMINE, K.; WIEMER, S. High b-values in the South Flank of Kilauea volcano, Hawaii: evidence for the distribution of magma below Kilauea's East rift zone. Journal of Vulcanology and Geothermal Research, v. 106, p. 23-37, 2001. 